\title{
Urgent Physiological Effects of the Vegetative Regulation of Adaptation Processes in Highly Skilled Athletes
}

\author{
Aleksanyants G.D. \\ Department of Anatomy and Sports Medicine \\ Kuban State University of Physical Education, Sports and \\ Tourism \\ Krasnodar, Russia \\ alexanyanc@mail.ru
}

\author{
Pogodin A.A. \\ Department of Sport and Physical Education \\ V.I. Vernadsky Crimean Federal University \\ Simferopol, Russia \\ andrei_mordvin@mail.ru.
}

\author{
Epishkin I.V. \\ Department of Sport and Physical Education \\ V.I. Vernadsky Crimean Federal University \\ Simferopol, Russia \\ igor.epishkin@gmail.com
}

\begin{abstract}
In the process of researching the autonomic nervous effects on the heart rhythm of different qualifications athletes in the context of urgent adaptation to physical exertion identified the atypical effects of sympathetic parasympathetic interactions in highly skilled athletes. The functional assessment of heart rate variability vegetative nervous regulation was carried out by the rheographic method using a bicycle ergometric test of the step-increasing load and the adequate statistical programs. The spectral, statistical and geometric parameters of heart rate variability characterizing sympatho-parasympathetic regulatory interactions in terms of the three threshold load conditions relative to the initial level of rest were analyzed. In the study of the heart rate variability under the conditions of the threshold load regimes in the anaerobic-aerobic threshold mode of work with the intensity of the heart rate in the range of 170185 beats per minute for athletes, having a relatively low athletic qualification revealed a typical physiological enhancement of sympathetic and decrease in parasympathetic modulating effects on heart rhythm. Highly qualified athletes (masters of sports and masters of sports of international class) have the effect of not reducing parasympathetic tonus under high-intensity load, which is more expressed in swimmers.
\end{abstract}

Keywords-autonomic nervous regulation; sympathetic parasympathetic influences; heart rate, urgent effects of adaptation of highly skilled athletes; threshold modes of work.

\section{INTRODUCTION}

The study of the neural regulatory mechanisms of adaptation is gaining an increasing role at the stages of sports training [7]. Balanced autonomic regulation of muscle activity allows the athlete to maximize the use of the body functional reserves, and also provides the necessary economization of the cardiovascular system functions during high-intensity muscular work [15]. An indicator of nervous regulatory optimality influences is heart rate $[1,2]$. It is known that the autonomic nervous regulation (ANS) of the heart rhythm is determined by the predominant influences of the sympathetic and parasympathetic divisions of the ANS, which are in complex interaction [6]. It is customary to assume that at rest the influence of both divisions on the heart is balanced, and with intense physical activity increases the activity of the sympathetic and decreases the activity of the parasympathetic division. It causes an increase in heart rate (HR). In this case, the mechanism of increasing $\mathrm{HR}$ is associated with a combination of two factors - elimination of the inhibitory effect of parasympathetic tone and enhance adrenergic regulation $[13,14]$. At the same time, a number of specialists in conditions of urgent adaptation to intensive loads along with an increase in sympathetic tone have noted in highly trained individuals not only the absence of the parasympathetic tone reducing effect, but also its enhancement $[8,9,10,11,12]$. Also attention is focused on a weak descriptive apparatus of such atypical regulatory effects functional evaluation [16]. In this regard, analysis of the vegetative regulatory algorithms for athletes of different qualifications can help identify the compensatory-adaptive reserves of the ANS, formed in the body as a result of long and intensive physical training. The goal was assessment of the adaptation processes vegetative regulation for athletes of different qualifications cyclic and game sports in terms of urgent adaptation to physical exertion.

\section{RESEARCH METHODOLOGY}

Swimmers and basketball players of 19-27 aged old $(n=53)$ with different sports qualifications were examined - first adult 
discharge $(n=18)$, candidates for master of sports (CMS, $\mathrm{n}=15$ ), master of sports and master of sports of international class (MS, MSIC, $n=20$ ). The study of Heart Rate Variability (HRV) was performed by the rheographic method using an eight-channel tetrapolar rheograph REOKOM Standard. To statistical analyzes were subjected the heart rate (HR, beats/min), the standard deviation of average NN intervals (SDANN), the square root of the sum of the differences of a consecutive series of cardiointervals (RMSSD, Ms), the number of pairs of cardiointervals with a difference of more than $50 \mathrm{~ms}$ in $\%$ to the total number of cardiointervals in the array (pNN50, n). The spectral analysis of HRV wave structure was used to determine the power (ms2) of different frequency characteristics waves: with high frequency vibrations (HF), low frequency oscillations (LF) and very low frequency oscillations (VLF). The coefficient of vagosympathetic interaction $(\mathrm{LF} / \mathrm{HF}, \%)$ was calculated by the ratio of HRV low-frequency and high-frequency components average values. Geometric analysis was used for determination of the mode indicators (Mo), the amplitude of the mode (AMo), the voltage index of the regulatory systems (VI). The indicators were studied at rest and with the ergometric step-increasing load [3], providing at least 5 minutes of work in threshold modes (W): aerobic (W1 - 60-75 $\mathrm{W}, \mathrm{HR}-120-130$ beats/min), aerobic-anaerobic (W2 - 120$150 \mathrm{~W}, \mathrm{HR}$ - 150-160 beats/min), anaerobic-aerobic (W3 -
180-220 W, HR - 170-185 beats/min). Studies were conducted in the preparatory period of the annual training process. The digital material was processed on a personal computer using the STATISTICA 10.0 software package. Verification of the normal distribution was carried out using the Shapiro-Wilk criterion. Next, the mean value $(\mathrm{X})$ of the studied quantities and the error of the arithmetic mean (sX) were calculated. Statistically significant differences were determined using Student's t-test with significance $\mathrm{p}<0,05$.

\section{RESULTS}

The study of athletes heart rhythm vegetative regulation in conditions of threshold load conditions allows to get an idea of the regulatory mechanisms, that support the physiological functions of SSS in muscle activity of different intensity and duration. Herewith a great importance has the range of fluctuations of vegetative indicators relative to the initial level. Changes in the parameters characterizing the sympathoparasympathetic regulatory interactions of athletes with highintensity load in the anaerobic-aerobic mode relative to the initial level of rest are presented in Table I. In these conditions, marked physiological changes in statistical values and the power of the spectral indices of HRV, characterizing vegetative reactions at the levels of sympathetic and parasympathetic regulation.

TABLE I. THE VALUES OF HRV INDICATORS OF ATHLETES IN THE INITIAL STATE (1) AND WHEN PERFORMING WORK IN THE ANAEROBIC-AEROBIC MODE (2)

\begin{tabular}{|c|c|c|c|c|c|c|}
\hline \multirow{2}{*}{ Options } & \multicolumn{2}{|c|}{1 adult level } & \multicolumn{2}{|c|}{ CMS } & \multicolumn{2}{|c|}{ MS, MSIC } \\
\hline & 1 & 2 & 1 & 2 & 1 & 2 \\
\hline \multicolumn{7}{|c|}{ values of swimmers statistics } \\
\hline SDANN, MS & $84,5 \pm 6,78$ & $57,8 \pm 3,57 * *$ & $87,6 \pm 2,05$ & $70,1 \pm 2,43 *$ & $137,7 \pm 3,67$ & $135,1 \pm 9,61$ \\
\hline RMSSD, MS & $32,2 \pm 1,09$ & $27,7 \pm 1,29 * *$ & $45,8 \pm 6,86$ & $36,6 \pm 6,16$ & $201,6 \pm 9,43$ & $208,6 \pm 8,13$ \\
\hline $\mathrm{pNN} 50, \%$ & $19,8 \pm 2,48$ & $11,8 \pm 2,84^{*}$ & $21,4 \pm 0,34$ & $16,5 \pm 1,92 *$ & $73,4 \pm 2,94$ & $64,3 \pm 1,45$ \\
\hline Mo, Ms & $750,3 \pm 37,8$ & $765,4 \pm 38,5$ & $816,7 \pm 13,9$ & $800,0 \pm 31,1$ & $1000,3 \pm 12,4$ & $1089,4 \pm 33,6$ \\
\hline AMo, $\%$ & $79,4 \pm 3,67$ & $122,5 \pm 12,1^{*}$ & $46,3 \pm 4,17$ & $51,8 \pm 2,80$ & $22,6 \pm 4,72$ & $28,80 \pm 2,82$ \\
\hline VI, srv. units & $176,7 \pm 14$ & $256,4 \pm 12,1 *$ & $114,3 \pm 7,9$ & $164,1 \pm 4,65^{*}$ & $75,3 \pm 0,81$ & $78,39 \pm 3,03$ \\
\hline \multicolumn{7}{|c|}{ spectral values of swimmers } \\
\hline VLF, $\mathrm{Ms}^{2}$ & $1007,8 \pm 16,9$ & $1246,9 \pm 19,6^{*}$ & $829,9 \pm 11,7$ & $1099,6 \pm 11,5^{*}$ & $965,9 \pm 16,7$ & $885,3 \pm 12,7$ \\
\hline $\mathrm{LF}, \mathrm{Ms}^{2}$ & $810,5 \pm 12,6$ & $1061,4 \pm 13,7$ & $1439,1 \pm 17,8$ & $1702,7 \pm 17,9^{* *}$ & $3689,4 \pm 16,9$ & $3944,6 \pm 16,0^{*}$ \\
\hline $\mathrm{HF}, \mathrm{Ms}^{2}$ & $540,5 \pm 18,23$ & $344,6 \pm 14,83 * *$ & $1336,7 \pm 9,0$ & $1203,9 \pm 10,8^{*}$ & $6142,6 \pm 19,4$ & $6125,5 \pm 36,2$ \\
\hline $\mathrm{LF} / \mathrm{HF}$ & $1,5 \pm 0,19$ & $3,08 \pm 0,26 * *$ & $1,06 \pm 0,11$ & $1,42 \pm 0,51 *$ & $0,62 \pm 0,06$ & $0,64 \pm 0,09$ \\
\hline \multicolumn{7}{|c|}{ values of statistical indicators of basketball players } \\
\hline SDANN, MS & $69,4 \pm 3,58$ & $46,8 \pm 4,01 *$ & $78,4 \pm 3,98$ & $56,4 \pm 4,38^{*}$ & $103,0 \pm 9,13$ & $88,0 \pm 3,72$ \\
\hline RMSSD, MS & $63,6 \pm 6,12$ & $34,5 \pm 2,57 * *$ & $92,4 \pm 6,40$ & $60,1 \pm 5,74 *$ & $165,65 \pm 7,5$ & $148,0 \pm 4,58$ \\
\hline pNN50,\% & $18,8 \pm 1,5$ & $15,1 \pm 1,3^{*}$ & $25,8 \pm 1,57$ & $19,4 \pm \pm 1,9 *$ & $67,8 \pm 1,08$ & $60,6 \pm 1,7 *$ \\
\hline Mo, MS & $725,0 \pm 28,12$ & $515,0 \pm 26,5^{*}$ & $1015,1 \pm 17,8$ & $835,0 \pm 19,6^{*}$ & $825,0 \pm 7,45$ & $800,0 \pm 39,51$ \\
\hline
\end{tabular}




\begin{tabular}{|c|c|c|c|c|c|c|}
\hline AMo, \% & $83,5 \pm 3,72$ & $131,4 \pm 6,18^{* * *}$ & $32,8 \pm 5,1$ & $66,2 \pm 4,4^{*}$ & $67,5 \pm 6,70$ & $61,8 \pm 3,70$ \\
\hline VI, srv. units & $202,5 \pm 7,7$ & $296,1 \pm 13,1^{*}$ & $164,4 \pm 6,02$ & $197,6 \pm 5,75^{*}$ & $74,3 \pm 6,01$ & $93,3 \pm 12,1$ \\
\hline \multicolumn{7}{|c|}{ values of spectral indicators of basketball players } \\
\hline VLF, мs & \multicolumn{7}{|c|}{$1286,2 \pm 19,3$} & $1503,1 \pm 17,1^{*}$ & $1176,8 \pm 13,5$ & $1417,4 \pm 16,3^{*}$ & $1100,3 \pm 15,5$ & $1255,5 \pm 19,4$ \\
\hline LF, Ms & $1398,0 \pm 13,7$ & $1666,0 \pm 16,3^{*}$ & $1327,4 \pm 15,6$ & $1555,0 \pm 19,2^{*}$ & $3530,3 \pm 13$ & $4030,3 \pm 12,5^{* *}$ \\
\hline HF, Ms & $681,3 \pm 12,0$ & $487,7 \pm 12,8^{*}$ & $1491,2 \pm 14,3$ & $1186,8 \pm 12^{*}$ & $3653,5 \pm 19,0$ & $3606,8 \pm 19,74$ \\
\hline LF/HF & $2,05 \pm 0,11$ & $3,03 \pm 0,12^{*}$ & $0,89 \pm 0,26$ & $1,31 \pm 0,25^{*}$ & $0,96 \pm 0,09$ & $1,11 \pm 0,11$ \\
\hline
\end{tabular}

Note - Reliability is indicated in relation to the initial state * $\mathrm{p}<0,05,{ }^{* *} \mathrm{p}<0,01,{ }^{* * *} \mathrm{p}<0,001$

\section{DISCUSSION}

So, the athletes with the qualifications of the first adult category and the CMS found a significant increase in the power of VLF and LF waves, a significant reduction in the power of $\mathrm{HF}$-waves, an increase in the vagosympathetic balance index LF/HF. It indicates the predominance of sympathetic influences and the strengthening of centralization in the managing of heart rhythm. With this type of vegetative regulation is determined the significant decrease in key variability index - SDANN and Mo indicator, increase AMo and VI. It indicates the relative tension of regulatory mechanisms.

The athletes MS and MSIC revealed a relatively high power of the HRV wave structure all the spectral parameters, a less power reduction of VLF- and HF- waves, namely, the lack of significant differences in the values of these indicators on the high-intensity threshold of load, that is, the absence of a reduction in parasympathetic influences in relation to the initial state. Under this type of regulation, a significant decrease in SDANN and Mo indices is not detected, indicators aMo and VI do not significantly increase, and LF/HF index decreases significantly, that according to the health criteria of Baevsky R.M. [2] corresponds to a high level of adaptation. It should be noted that in the group of highly qualified athletes specializing in cyclic sports (swimming in open water) and including athletes MSIC the marked variant of regulation is more expressed regarding game types (basketball players). The magnitude of the swimmers HF-waves power during the load was $6125,5 \pm 36,2$ ms2 in relation to the indicator $6142,6 \pm 19,4$ Ms2 ( $>00,05)$, and of the basketball players was $3686,8 \pm 19,74 \mathrm{~ms} 2$ and $3606,8 \pm 19,74 \mathrm{~ms} 2(\mathrm{p}>0,05)$. A similar trend has been identified with regard to the magnitude of VLF waves power, which representatives of swimming during loading is $885,3 \pm 12,7 \mathrm{Ms} 2$, and in the initial state is $965,9 \pm 16,7 \mathrm{~ms} 2 \quad(\mathrm{p}>0,05)$, whereas representatives of basketball is $1255,5 \pm 19,4 \mathrm{~ms} 2$ and $1100,3 \pm 15,5 \mathrm{Ms} 2$ ( $>0,05)$. That is in the absence of significant differences in the average values of the spectral parameters power values in the state of rest and load, these indicators swimmers under load conditions are reduced to a lesser extent. Also, highly qualified swimmers have relatively low indicators of average values of the LF/HF and VI indices - swimmers have $78,39 \pm 3,03$ and $75,3 \pm 0,81$ srv. units $(p>0,05)$, and basketball players $-93,3 \pm 12,1$ and 74,3 $\pm 6,01$ srv. units ( $>0,05)$. Such statistics of the highly skilled athletes, as RMSSD and pNN50, characterizing the activity of the parasympathetic division of the ANS during load, also significantly unchanged. In this way, for athletes with a high level of qualification characteristic is the variant of the nervous regulation in which there is no effect of reducing the parasympathetic tone with a threshold heart rate of 170-185 beats/min. At first sight, the effect, that we noticed, is inconsistent with the idea of adaptation regulatory mechanisms that increase HR. However, in a number of scientific publications it has been shown that a decrease in parasympathetic tone during load can occur at different HR, depending on the functional level of SSS. So, the analysis of cardiointervals variability mathematical model obtained during stress testing [11], allowed to determine the instantaneous HR point at which the variability decreases and cardiac muscle escapes from parasympathetic control. For untrained persons this point is recorded at 135 beats $/ \mathrm{min}$, for highly trained is recorded at 170 beats/min, that is in fact, with anaerobic-aerobic threshold load, which in the opinion of the author is a favorable sign of adaptation. At the same time, a similar effect, noted by the authors [9] of marathoners, is considered as a violation of the autonomic regulation of heart rhythm and a sign of failure of cardiac mechanisms and in particular the Frank-Starling mechanism, that with this option for the urgent adaptation to a long-term stressed load can lead to a state of fibrillation. Obviously, there are threshold levels of HR at which the parasympathetic control of the heart muscle is safe and does not cause the severe functional myocardial disorders [4]. Exceeding this threshold requires a transition to sympathetic and adrenergic regulation, providing the increased myocardial contractility with the developing phenomena of hypoxia load. This assumption is supported by the established patterns of urgent adaptation of highly skilled athletes and the concept of antihypoxic functional systems (AFS) [5], which achieve an antihypoxic effect due to the intensification of effector activity (increase HR and stroke volume of the heart) not increasing, but reducing energy waste. As leading components of the AFS act the brake systems of the central nervous system and relaxation processes of nervous and muscular regulation. Probably, the established effect of a decrease absence in the parasympathetic tone under high-intensity load for highly skilled athletes is associated with a high activity of the antihypoxic adaptation mechanism. 
[7] Kuznetsova Z., Kuznetsov A., Mutaeva I., Khalikov G., Zakharova A. Athletes preparation based on a complex assessment of functional state. Proceedings of the $3^{\text {rd }}$ International Congress on Sport Sciences Research and Technology Support. 2015.

1. Under conditions of anaerobic-aerobic threshold work for athletes of relatively low qualification level (first discharge, CMS) is characteristic variant of the autonomic regulation of heart rhythm, which increases the activity of sympathetic and decreases the activity of parasympathetic modulating effects on the myocardium.

2. In athletes with a high level of qualification (MS, MSIC), the effect of the absence of a decrease in parasympathetic influences on the heart rate was revealed with greater severity in swimmers at a threshold heart rate of 170185 beats/min.

\section{References}

[1] Akhmetov, S.M., Pogodina S.V., Manolaki V.G., Aleksanyants G.D. Physiological features and reserves of the cardiovascular system of professional athletes in the pre-devolution period. Man. Sport. The medicine. 2018, pp. 46-54.

[2] Baevsky, R.M. The concept of physiological norm and health criteria. Russian physiological journal named by Sechenov. 2003, vol. 89, 4, pp. 473-487.

[3] Belotserkovsky, Z. B. Ergometric and cardiological criteria for physical performance in athletes. Moscow: Soviet Sport. 2005, 312.

[4] Vanyushin, Yu. S., Khairullin R.R., Rakhimov M.I. Threshold of adequate hemodynamic response in athletes during physical activity of increasing power. Theory and Practice of Physical Culture. 2016, 9, pp. 53-55.

[5] Vysochin, Yu. V., Denisenko Yu.P. Modern ideas about the physiological mechanisms of the urgent adaptation of the body of athletes to the effects of physical activity. Theory and Practice of Physical Culture. 2002, 7, pp. 2-5.

[6] Kuznetsov S.A., Kuznetsova Z.M. The history of Paralympic sport development in the world. Pedagogico-psychological and medicobiological problems of physical culture and sport. 2014, 2(31), pp. 3440. DOI: 10.14526/00_1111_06.

[8] Gorst, V.R., Gorst N.A., Polukova M.V. Disagreement of the rhythms of the cardiovascular and respiratory systems with maximum physical exertion. Astrakhan Medical Journal. 2011, vol. 6, 2, pp. 242-244.

[9] Ivanova, N. V. Evaluation of the functional state of the cardiorespiratory system of athletes with different specificity of muscular activity in the competitive period of preparation. Bulletin of sports science. 2011, 1, pp. 64-68.

[10] Kudrya, O.N., Belova L.E., Kapilevich L.V. Adaptation of the cardiovascular system of athletes to loads of different directions. Tomsk State University Bulletin. 2012, 356, pp.162-166.

[11] Meerson, F.Z., Pshennikova M.G. Adaptation to stress situations and physical loads. M.: Medicine. 1988, 256.

[12] Pogodina, S.V., Yuferev V.S., Aleksanyants G.D. Physiological features of the cardiovascular system in male athletes at 17-46 years old. Bulletin of Adygea State University. Series 4: Natural Mathematical and Technical Sciences. 2015, 1(154), pp. 36-48.

[13] Pokhachevsky, A. L. Evaluation of the functional state of the cardiac rhythmogram with bicycle ergometry. Human Physiology. 2007, vol. 33, 6, pp. 117-119.

[14] Aleksanyants, G., Pogodina S., Yuferev V., Epishkin I. Signal indicators of regulatory changes in the respiratory system under physiological deviation conditions. Bulletin of the Georgian national academy of sciences. 2018, vol. 12, 4, pp. 13-19.

[15] Buchheit, M., Chivot A., Parouty J. et al. Monitoring endurance running performance using cardiac parasympathetic function. European Journal of Applied Physiology. 2010, vol. 108, pp. 1153-1167.

[16] Carter, J.R., Ray C.A. Sympathetic neural adaptations to exercise training in humans. Autonomic Neuroscience. 2014, vol. 188, pp. 36-43.

[17] Eckberg, D.L. Sympathovagal balance: a critical appraisal. Circulation. 1997, 96, pp. 3224-3232.

[18] Goldberger, J.J., Kiet Le F., Lahiri M., et al. Assessment of parasympathetic reactivation after exercise. Am. J. Physiol. Heart Circ. Physiol. 2006, vol. 290, pp. H2446-H2452. 